\title{
Interaction between CETP Polymorphism and Dietary Insulin Index and Load on Cardiovascular Risk Factors: A Cross-Sectional Study of Diabetic adults
}

Faezeh Abaj

Tehran University of Medical Sciences

Masoumeh Rafiee

Isfahan University of Medical Sciences

Fariba Koohdani ( $\sim$ fkoohdan@tums.ac.ir)

Tehran University of Medical Sciences

\section{Research Article}

Keywords: CETP Taq1B polymorphism, T2DM, Cardiovascular diseases, Dietary insulin indices, Personalized nutrition

Posted Date: March 6th, 2021

DOI: https://doi.org/10.21203/rs.3.rs-286557/v1

License: (c) (i) This work is licensed under a Creative Commons Attribution 4.0 International License. Read Full License

Version of Record: A version of this preprint was published at Scientific Reports on August 5th, 2021. See the published version at https://doi.org/10.1038/s41598-021-95359-y. 


\section{Abstract \\ Background}

The objectives were to investigate the effect of the interaction between CETP Taq1B polymorphism, Dietary Insulin Index and Insulin Load (DII and DIL) on cardiovascular risk factors among diabetic patients.

\section{Methods}

In this cross-sectional study, blood samples were collected from 220 patients. DIL and DII were obtained via validated FFQ. CETP Taq1B polymorphism was genotyped by the PCR-RFLP method.

\section{Results}

The highest BMI $(P=0.08)$ and WC $(P=0.01)$ values were observed in B2B2 genotype with the highest adherence to DII. Patients with B1B1 genotype who were in the highest DIL tertile had lower LDL/HDL $(P=0.001), T G(P=0.03)$, and higher HDL $(P=0.02)$. The highest SOD $(P=0.01), P G F 2 a(P=0.04), C R P(P=$ 0.02), and IL-18 ( $P=0.06)$ was observed in B2B2 genotype carriers with the highest DIL adherence. Individuals with B2B2 genotype in the highest tertile of DII had higher $\operatorname{CRP}(P=0.04)$, TAC $(P=0.01)$, SOD $(P=0.02)$, and PGF2a $(P=0.02)$. B1B1 homozygotes who were in the highest tertile of $D I I$ had lower lipid profile TG $(P=0.02)$, LDL $(P=0.08)$, and $L D L / H D L<0.001$.

\section{Conclusion}

Based on the current study, it may be proposed that CETP Taq1B polymorphism can be associated with CVD risk factors in diabetic patients with high adherence to insulin indices, including DII and DIL

\section{Introduction}

Type 2 diabetes mellitus (T2DM) is a metabolic disorder that occurs with high serum glucose concentrations, caused by deficiencies in insulin secretion or function (1). T2DM is a worldwide pandemic that imposes an enormous burden on public health, also being an elevated risk factor for cardiovascular disease (CVD). Patients with CVD have more than a two-to-four-fold increased risk of death versus non-CVD patients (2). As a multifactorial condition, T2DM and CVD are also determined by environmental conditions, hormonal factors, and genetic variations, which have been related to $50 \%$ of incidence (3). Recently, emerging data suggest that one of the main genetic targets is the cholesteryl ester transfer protein (CETP), which plays a crucial role in regulating lipid metabolism (4).

CETP is involved in the reverse cholesterol transport process by mediating the exchange of cholesteryl esters (CE) and triglycerides (TGs) from high-density lipoprotein cholesterol (HDL) to low-density lipoprotein (LDL) in peripheral tissues to the hepatocytes (5). The CETP gene is very polymorphic in humans, such that rs708272 (also called Taq1B) has been widely studied among CETP polymorphisms (6). According to various studies, there is a relationship between rs708272 and various CVD risk factors including T2DM, hypertension, dyslipidemia, and low HDL, although the evidence is controversial: other studies have indicated a lack of such associations (6-8). The major lifestyle factor contributing to DM and CVD, however, has been shown to be dietary pattern (9).

It seems that foods can induce postprandial insulin secretion and affect the management of hyperlipidemia, cardiac artery disease (CAD), obesity, and DM (10). Most previous studies have concentrated on dietary glycemic index (GI) and load (GL) as indices of carbohydrate quality and quantity, to demonstrate the insulinogenic effects of foods (11). Although GI and GL indicate the effect of carbohydrates on blood glucose concentrations, these indices are not always accurate in reporting insulin response (12). DII and DIL can more accurately predict insulin response to overall food consumption, determining how insulinogenic dietary properties can be mediated by various factors such as fructose, certain amino acids, and fatty acids (13). Prior studies have demonstrated that DII and DIL are more appropriate (than GI, GL, and total carbohydrate consumption) for investigating the relationship between insulin concentrations and the progress of chronic disorders (14). Relatively few studies in the literature have investigated the correlation between DII, DIL, and CVD risk factors, and the correlation remains unclear within current nutrition research (15). Several studies which showed inconsistent results may be explained by differences in genetic variations (15). Therefore, the interaction between genetics and diet (in terms of both a nutrigenetic and nutrigenomic approach) is essential in assessing these associations, which are still not well understood (16). Some findings revealed that the CETP polymorphism interacts with dietary carbohydrate intake on metabolic factors, such as hypertension, dyslipidemia, obesity, insulin resistance (IR) and DM (17).

To the authors' knowledge, there has been no previous evaluating the interaction between the DII, DIL and CETP polymorphism towards CVD risk factors. Therefore, the present study aimed to evaluate the effects of interaction between CETP TaqB1 polymorphism and the DII and DIL insulin indices on CVD risk factors in T2DM patients.

\section{Results}

In the current study, 220 patients with T2DM were evaluated. The genotype distributions were within HWE (P-value $>0.05)$. A statistical analysis of the basic information of patients, among DII and DIL tertiles, are presented in Table 1. An individual with higher adherence to DIL $(P=0.001)$ and DII $(P=0.006)$ was 
more likely to be male. Subjects with a higher DIL tertile presented greater energy intake values $(P=0.001)$. Patients in the highest tertile of DIL $(P=0.01)$ and DII $(P=0.01)$ were more likely to have lower ghrelin concentrations.

Figure 1 and Fig. 2 show the interactions between CETP TaqB1 polymorphism and DIL and DII on anthropometric indices and several biochemical markers.

CETP TaqB1 polymorphism and DIL interactions were significant in terms of LDL/HDL $(P=0.001)$, TG $(P=0.03)$, and HDL $(P=0.02)$ : carriers of the B1B1 genotype who were in the highest tertile of DIL had lower LDL/HDL, TG, and higher HDL.

Interactions between CETP TaqB1 polymorphism and DIL were significant in terms of WC $(P=0.005)$ : carriers of B2B2 genotype who were in the last tertile of DIL had higher WC. Also, CETP TaqB1 polymorphism and DIL interactions were significant in terms of SOD $(P=0.01), P G F 2 a(P=0.04)$, CRP $(P=0.02)$, and $I L-$ $18(P=0.06)$; higher SOD, PGF2a, CRP, and IL-18 values were observed in B2B2 genotype carriers with the highest adherence to DIL.

Further, a significant interaction between CETP TaqB1 and DII was shown in terms of BMI $(P=0.08)$ and WC $(P=0.01)$, where the highest BMI and WC were observed in B2B2 genotype carriers with the highest adherence to DII. Also, a significant interaction between CETP TaqB1 and DII was shown in terms of CRP $(P=0.04)$, TAC $(P=0.01)$, SOD $(P=0.02)$, and PGF2a $(P=0.02)$ individuals with B2B2 genotype in the last tertile of DII had higher CRP, TAC, SOD, and PGF2a.

A significant interaction between CETP rs708272 and DIl was shown in terms of TG $(P=0.02)$, LDL/HDL $(P<0.001)$, and LDL $(P=0.08)$ : B1B1 homozygotes in the highest tertile of DII had lower lipid profiles.

\section{Discussion}

To the authors' knowledge, this was the first study investigating the interaction between CETP Taq1B polymorphism and DII and DIL on CVD risk factors in T2DM patients. The key findings of the current study were the significant interaction effect of CETP rs708272 polymorphism with DII and DIL on obesity indices (WC and BMI), lipid profiles (TG, HDL, LDL/HDL), inflammatory markers (IL-18, CRP, and PGF2a), and antioxidant markers (TAC and SOD) in T2DM patients.

T2DM patients have an elevated risk of CVD. Additionally, CETP is a key gene related to CVD pathogenesis (18). The relation between the CETP Taq1B polymorphism and the risk factors of CVD in patients with T2DM has been evaluated in previous studies. A significant interaction between CETP Taq1B polymorphism and DII, DIL was demonstrated in relation to obesity indices, including WC and BMI. CETP Taq1B polymorphism was found to be able to increase the effects of DII and DIL on obesity. In the present study, B2B2 genotype carriers in the last tertile of DII and DIL were more obese. Although several studies have reported an association between DIL and DII scores and obesity $(19,20)$, the interaction between DII and DIL with CETP polymorphism on obesity was not evaluated in previous reports.

DII and DIL may represent insulin concentrations in response to consuming certain foods, and are perhaps more appropriate than dietary GI and GL in assessing the relation between insulin exposure and metabolic disorders (21). It is proposed that DIL and DII have a direct and indirect effect on obesity. A high score of DIL and DII may lead to greater body fat formation by promoting pre-adipocytes differentiation and proliferation to adipocytes (19, 20). Besides, a high score may cause reductions in insulin sensitivity and lipolysis, while increasing insulin growth factor-1 (IGF-1) and insulin secretion levels (22, 23). Experimental studies have shown that a high stimulation of IGF-1 on pre-adipocytes proliferation could be associated with body fat accumulation (24). IGF-1 increased glucose uptake and glucose oxidation in adipocytes, elevated lipogenesis, and inhibited lipolysis in cells (25). According to some studies, insulinogenic foods result in a higher risk of insulin resistance and DII/DIL, independent from potential risk factors (26). Dietary pattern interventions, such as restricting insulinogenic foods, have a key role in the management of IR and its metabolic disorders (27). CETP is a protein synthesized by various tissues, especially adipose tissue in humans, which leads to the storing of CEs in adipocytes, resulting in the formation of fatty deposits (28). Results have suggested a potential pathway relating CETP polymorphisms with an elevated risk of obesity and obesity-related diseases (29). Additionally, there is a linear association between CETP activity and insulin resistance in obese T2DM patients; high CETP activity also contributes to high insulin secretion (30). Therefore, high DII/DIL score and CETP polymorphism may act synergistically to elevate a patient's susceptibility to insulinemic spikes, which relate to obesity.

The present findings are consistent with previous studies that have reported elevated plasma CETP activity in obese participants. Dullaart et al. demonstrated that plasma CETP activity was increased in obese subjects. Also, CETP activity was related to BMI and plasma C-peptide (31). Arai et al. revealed that CETP activity and protein levels were elevated in obese participants (32). In contrast to the present study, however, Heilbronn et al. reported that BMI was higher in obese B1B2 participants, compared to obese subjects with B1B1 and B2B2 (33). Maroufi et al. reported that Taq1B polymorphisms had no effect on related metabolic syndrome parameters, including WC (34). Also, some studies have shown that CETP polymorphism is not associated with anthropometric parameters $(35,36)$. These inconsistencies seem due to an important role of gene-diet interactions. It has been shown that relations between CETP Taq1B and anthropometric indices can be population-specific, and consequently are regulated by environmental factors, especially dietary factors (37).

The present results also demonstrated a significant interaction among CETP Taq1B polymorphism and DII/DIL in relation to inflammatory factors. The highest IL-18, CRP, and PGF2a were observed in the B2B2 genotype carriers with the highest adherence to DIL and DII. As indicated in the findings, there was a significant interaction effect between Taq1B CETP polymorphism and DII/DIL in association with anthropometric indices. Numerous findings have reported obesity as causing chronic low-grade inflammatory disorder, contributing to the progression of T2DM and CVD (38). In these conditions, human adipose tissue secretes a high level of inflammatory markers, including IL-18, CRP, and PGF2a (39).

A further novel finding is the significant interaction between CETP Taq1B polymorphism and DII/DIL towards antioxidant markers, including TAC and SOD. CETP Taq1B polymorphism was able to inverse the effect of DII and DIL on oxidant status, so that the highest TAC and SOD was observed in the B2B2 genotype with the highest adherence to DIL and DII. However, while there is no available study about the relation between DIL/DII with antioxidant status, several studies have reported that insulin concentrations and insulin resistance lead to an imbalance between oxidant and antioxidant systems, a condition

Page $3 / 10$ 
known as oxidative stress (40). In recent years, oxidative stress has been implicated in T2DM pathogenesis by producing excessive free radicals, decreasing glutathione, vitamin E, vitamin C, and via reduced antioxidant enzyme activity, such as SOD and TAC (41). SOD and TAC lead to the conversion of superoxide radicals into hydrogen peroxide, and decrease oxygen toxicity (41). It seems that CETP Taq1B polymorphism can invert the effect produced by insulin.

However, the reason for this difference is not apparent, and further studies are warranted to evaluate the mechanism of action.

Finally, another promising finding was the significant interaction between CETP Taq1B polymorphism and DII/DIL with lipid profile markers, including TG, HDL, and LDL/HDL. The lowest TG and LDL/HDL, and the highest HDL, were observed in the B1B1 genotype carriers with the highest adherence to DIL and DII. Although several studies have shown a relationship between DII/DIL scores and lipid profiles, the interaction between DII/DIL and CETP polymorphism on lipid profiles had not been evaluated in previous studies.

Additionally, prior research has shown that the B1B1 genotype is associated with a better response to nutritional interventions, compared with carriers of B2 alleles. Previous research showed a significant interaction, where B1/B1homozygotes had a lower TG/HDL ratio after a kiwifruit intervention, compared to a control diet, while B2 carriers were not affected (42). Nahid Ramezani-Jolfaie et al. revealed that, for diabetic patients, dietary oil treatments would be more helpful (lower LDL; HDL, TG; HDL, TC; HDL, Insulin, and HOMA-IR) among subjects with B1B1 alleles than among B2 allele carriers (43).

Some authors have also suggested that subjects with B1B1 allele of CETP polymorphism showed a better response in regards to high carbohydrate dietary interventions. Overall these findings are in accordance with findings reported by Juan Dua et al., demonstrating that males with CETP Taq1B B1B1 allele have higher apo A-I and HDL concentrations after following a high carbohydrate and low fat (HC/LF) diet for 6 days (44). Perez et al. reported that carriers of B2 alleles who consumed sucrose as more than $5 \%$ of total kcal/day had higher TC and LDL serum levels, compared to B1B1 homozygotes (45).

A series of recent studies have indicated that plasma CETP activity decreased by hyperinsulinemia condition in healthy subjects, but not in diabetic patients (46). Siewert et al. suggested that insulin has a direct influence on CETP, but in IR conditions, this particular insulin action may be diminished (47). Therefore, it is suggested that diabetic patients with B1B1 allele may be counteracted with CETP activity and HDL plasma reduction.

Several studies have been shown that CETP is able to transfer HDL cholesterol esters to Apolipoprotein B (ApoB), including VLDL, remnants of VLDL, and LDL. Due to an important role of CETP polymorphisms in lipids metabolism, it is considered that genetic polymorphism in the CETP gene is related to CVD risk factors by changing serum lipid profiles $(48,49)$. The inconsistency may be due to distinct populations; the studies were conducted among different gender, ethnic, and geographic populations, with a variety of habitual dietary patterns and various underlying diseases.

\section{Conclusion}

Based on the current study, it could be proposed that CETP polymorphism may be associated with CVD risk factors in T2DM patients with high adherence to insulin indices, including DII and DIL. This conclusion illustrates that the CETP Taq1B B1 allele could counteract the CVD risk induced by high DII and DIL. This could be critical for clinical diagnosis and gene-based therapy. Due to the limited nature of the study, further research is warranted to assess the effects on other populations.

\section{Methods}

\section{Study Population}

A cross-sectional study was designed with 220 diabetic patients in Tehran, Iran, who had participated in a large study conducted previously (50). All criteria including inclusion and exclusion criteria, demographic data, dietary intake (147-item FFQ), physical activity questionnaire (METs), and anthropometric variables in the current study were extracted from the authors' previous research. All study subjects gave their written informed consent and the

study was conducted based on the Declaration of Helsinki, and Ethics Committee of the Tehran University of Medical Sciences approved the protocol (no. 15060).

\section{General, Anthropometric and Physical Activity Assessments}

Information such as age, disease history, and medication was collected from each participant by questionnaire. Anthropometric measurements, including height $(\mathrm{m})$ and weight $(\mathrm{kg})$ were evaluated without shoes, and using a digital scale; waist circumference (WC) was evaluated at the narrowest part of the abdomen. Finally, body mass index (BMI) was computed by dividing weight (kg) by height squared (meters). Physical activity was measured by the short-form International Physical Activity Questionnaire (IPAQ) (51).

\section{Biochemical Assessments}

All blood samples were collected after 12-14 hours of fasting at the Nutrition and Genomics Laboratory at TUMS. All samples were centrifuged and stored at $-80^{\circ} \mathrm{C}$ until analysis. Inflammation markers, including IL-18, PGF2, and CRP concentrations, were measured by using an enzyme-linked immunosorbent assay (ELISA). TG, HDL, total cholesterol, and LDL serum levels were determined by enzymatic method (Pars Azmun Co., Tehran, Iran). Superoxide dismutase (SOD) enzymatic activity was measured using the colorimetry method (Cayman Chemical Company, USA). Spectrophotometry was used to detect total antioxidant capacity (TAC).

\section{Dietary Assessment}


A validated 147-item food-frequency questionnaire (FFQ) was used to obtain a common dietary intake for patients (52). The FFQ contained 147 food items with standard portion sizes commonly used by Iranians, based upon on measurements widely used in the community (e.g. number of slices for bread, glasses for drinks, plates for rice, etc.) (53). The portion sizes in the FFQ were converted into amounts of foods consumed. An expert interviewer carried out the FFQ and recorded participant responses. All reported consumption frequencies were converted to grams per day using household measures. The daily consumption of nutrients and total energy were calculated for each subject by using the Iranian food composition table (FCT) and the United States Department of Agriculture (USDA) guidelines.

\section{Assessment of DIL and DII}

DIl was based on prior studies conducted by Brand-Miller (54). DIl calculates the incremental insulin area under the curve over $2 \mathrm{~h}$, in response to the consumption of a 1000-kJ portion of the test food, then divided by the area under the curve after ingestion of a 1000-kJ portion of the reference food (54-56). In the current study, food items in the FFQ and the Brand-Miller study were matched in terms of energy content, carbohydrates, fiber, protein, and fat. To evaluate the average DIL, the insulin load from each food over the past year was calculated by the formula:

Insulin load of food $=$ Insulin index of food $\times$ energy content of food $(\mathrm{kcal} / \mathrm{d})$

By summing up the insulin load from each food, DIL was determined. The DIl of each food was computed by dividing DIL by total energy intake.

\section{Genotyping}

DNA genotyping was carried out by the salting-out extraction method, as previously published (57). CETP polymorphism (rs708272) was genotyped by the polymerase chain reaction-restriction fragment length polymorphism (PCR-RFLP) method. The PCR technique was employed by using primer (F:50CACTAGCCCAGAGAGAGGAGTG-30; R: 50 -TGAGCCCAGCCGCACACTAAC-30). 2\% agarose gel electrophoresis was used to analyze the product.

\section{Statistical Analysis}

All data were analyzed using IBM SPSS Statistics (version 25; SPSS Inc., IL). Significance level was considered P<0.1 for interaction analysis, and normality was analyzed by Kolmogorov Smirnov test.

Adherence to Hardy-Weinberg equilibrium (HWE) was determined by using the chi-square test. The means of variables across the tertiles of DII and DIL were expressed as means \pm SDs. Crude means between three genotypes (B1B1, B1B2, and B2B2) groups were compared using a one-way ANOVA test. The interaction between CETP rs708272 polymorphism and insulin indices (DII and DIL) on CVD factor (BMI, WC, HDL, LDL, LDL/HDL, T. Chol, TG, CRP, IL-18, TAC, SOD, PGF2a) was performed by a generalized linear regression model (GLM) in both the crude and adjusted models. In all interaction analyses, age, gender, physical activity, smoking, alcohol consumption, and familial history of diabetes were matched in the adjusted model.

\section{Declarations}

\section{ACKNOWLEDGMENTS}

The authors would like to thank authorities in Tehran University of Medical Sciences for financial support of the study.

\section{Funding sources}

This research was supported by Tehran University of Medical Sciences, Tehran, Iran.

\section{Author contributions}

FA contributed to conception, design, data analyses, data interpretation, and manuscript drafting. FK and MR supervised the study. All authors approved the final manuscript for submission

\section{Conflicts of interest}

None of the authors declared any personal or financial conflicts of interest.

\section{References}

1. Mahmoud JI. Osteoporosis in diabet patient. 2018.

2. Wittwer JA, Golden SH, Joseph JJJCCRR. Diabetes and CVD Risk: Special Considerations in African Americans Related to Care. 2020;14(10):1-14.

3. Buraczynska M, Ksiazek K, Zukowski P, Grzebalska AJDr, practice c. Interleukin-18 gene polymorphism and risk of CVD in older patients with type 2 diabetes mellitus. 2016;121:178-83.

4. Blauw LL, Li-Gao R, Noordam R, de Mutsert R, Trompet S, Berbée JF, et al. CETP (cholesteryl ester transfer protein) concentration: a genome-wide association study followed by Mendelian randomization on coronary artery disease. 2018;11(5):e002034.

5. Millwood IY, Bennett DA, Holmes MV, Boxall R, Guo Y, Bian Z, et al. Association of CETP gene variants with risk for vascular and nonvascular diseases among Chinese adults. 2018;3(1):34-43. 
6. Semaev S, Shakhtshneider E, Orlov P, Ivanoshchuk D, Malyutina S, Gafarov V, et al. Association of RS708272 (CETP Gene Variant) with Lipid Profile Parameters and the Risk of Myocardial Infarction in the White Population of Western Siberia. 2019;9(11):739.

7. El-Lebedy DJCd. Interaction between endothelial nitric oxide synthase rs1799983, cholesteryl ester-transfer protein rs708272 and angiopoietin-like protein 8 rs2278426 gene variants highly elevates the risk of type 2 diabetes mellitus and cardiovascular disease. 2018;17(1):97.

8. Raina JK, Sharma M, Panjaliya RK, Dogra V, Bakaya A, Kumar PJBCD. Association of ESR1 (rs2234693 and rs9340799), CETP (rs708272), MTHFR (rs1801133 and rs2274976) and MS (rs185087) polymorphisms with Coronary Artery Disease (CAD). 2020;20(1):1-13.

9. Mirmiran P, Bahadoran Z, Vakili AZ, Azizi FJAP, Nutrition, Metabolism. Western dietary pattern increases risk of cardiovascular disease in Iranian adults: a prospective population-based study. 2017;42(3):326-32.

10. Frendo-Cumbo S, MacPherson RE, Wright DCJPr. Beneficial effects of combined resveratrol and metformin therapy in treating diet-induced insulin resistance. 2016;4(15):e12877.

11. Vega-López S, Venn BJ, Slavin JLJN. Relevance of the glycemic index and glycemic load for body weight, diabetes, and cardiovascular disease. 2018;10(10):1361.

12. Eleazu COJAhs. The concept of low glycemic index and glycemic load foods as panacea for type 2 diabetes mellitus; prospects, challenges and solutions. 2016;16(2):468-79.

13. Yari Z, Behrouz V, Zand H, Pourvali KJCdr. New insight into diabetes management: from glycemic index to dietary insulin index. 2020;16(4):293-300.

14. Sadeghi O, Hasani H, Mozaffari-Khosravi H, Maleki V, Lotfi MH, Mirzaei MJJotAoN, et al. Dietary Insulin Index and Dietary Insulin Load in Relation to Metabolic Syndrome: The Shahedieh Cohort Study. 2020.

15. Lee DH, Giovannucci EL, Tabung FKJBJoN. Insulin-related dietary indices predict 24-h urinary C-peptide in adult men. 2020:1-8.

16. Comerford KB, Pasin GJN. Gene-dairy food interactions and health outcomes: A review of nutrigenetic studies. $2017 ; 9(7): 710$.

17. AL-Azzawie AF, Al-sugmiany RZ, Salih NAJEJoB. Cholesteryl ester transfer protein Taq1B gene polymorphism in some Iraqi patients with lipid disorders. 2019;13(1):253-8.

18. Masson W, Lobo M, Siniawski D, Huerín M, Molinero G, Valero R, et al. Therapy with cholesteryl ester transfer protein (CETP) inhibitors and diabetes risk. 2018;44(6):508-13.

19. Mirmiran P, Esfandiari S, Bahadoran Z, Tohidi M, Azizi FJJoD, Disorders M. Dietary insulin load and insulin index are associated with the risk of insulin resistance: a prospective approach in tehran lipid and glucose study. 2015;15(1):23.

20. Anjom-Shoae J, Keshteli AH, Sadeghi O, Pouraram H, Afshar H, Esmaillzadeh A, et al. Association between dietary insulin index and load with obesity in adults. 2019:1-13.

21. Nimptsch K, Brand-Miller JC, Franz M, Sampson L, Willett WC, Giovannucci EJTAjocn. Dietary insulin index and insulin load in relation to biomarkers of glycemic control, plasma lipids, and inflammation markers. 2011;94(1):182-90.

22. Joslowski G, Goletzke J, Cheng G, Günther A, Bao J, Brand-Miller J, et al. Prospective associations of dietary insulin demand, glycemic index, and glycemic load during puberty with body composition in young adulthood. 2012;36(11):1463-71.

23. Joslowski G, Halim J, Goletzke J, Gow M, Ho M, Louie JC-Y, et al. Dietary glycemic load, insulin load, and weight loss in obese, insulin resistant adolescents: RESIST study. 2015;34(1):89-94.

24. la Fleur S, Luijendijk M, Van Rozen A, Kalsbeek A, Adan RJljoo. A free-choice high-fat high-sugar diet induces glucose intolerance and insulin unresponsiveness to a glucose load not explained by obesity. 2011;35(4):595-604.

25. Assefa B, Mahmoud AM, Pfeiffer AF, Birkenfeld AL, Spranger J, Arafat AMJOm, et al. Insulin-like growth factor (IGF) binding protein-2, independently of IGF-1, induces GLUT-4 translocation and glucose uptake in 3T3-L1 adipocytes. 2017;2017.

26. Kopp WJMh. Role of high-insulinogenic nutrition in the etiology of gestational diabetes mellitus. 2005;64(1):101-3.

27. Bando H, Ebe K, Muneta T, Bando M, Yonei YJJoC, Endocrinology E. Proposal for insulinogenic index (IGI)-carbo70 as experimental evaluation for diabetes. 2017;1(1):102.

28. Salerno A, Silva T, Amaral M, Alberici L, Bonfleur M, Patricio P, et al. Overexpression of apolipoprotein CIII increases and CETP reverses diet-induced obesity in transgenic mice. 2007;31(10):1586-95.

29. Sandhofer A, Tatarczyk T, Laimer M, Ritsch A, Kaser S, Paulweber B, et al. The Taq1B-variant in the Cholesteryl Ester-Transfer Protein Gene and the Risk of Metabolic Syndrome. 2008;16(4):919-22.

30. Xin G, Yang G, Hui LJDr, practice c. Study to assess whether waist circumference and changes in serum glucose and lipid profile are independent variables for the CETP gene. 2014;106(1):95-100.

31. Dullaart R, Sluiter W, Dikkeschei L, Hoogenberg K, Tol AVJEjoci. Effect of adiposity on plasma lipid transfer protein activities: a possible link between insulin resistance and high density lipoprotein metabolism. 1994;24(3):188-94.

32. Arai T, Yamashita S, Hirano K-i, Sakai N, Kotani K, Fujioka S, et al. Increased plasma cholesteryl ester transfer protein in obese subjects. A possible mechanism for the reduction of serum HDL cholesterol levels in obesity. 1994;14(7):1129-36.

33. Heilbronn LK, Noakes M, Clifton PJA. Association between HDL-cholesterol and the Taq1B polymorphism in the cholesterol ester transfer protein gene in obese women. 2002;162(2):419-24.

34. Maroufi NF, Farzaneh K, Alibabrdel M, Zarei L, Cheraghi O, Soltani S, et al. Taq1B polymorphism of cholesteryl ester transfer protein (CETP) and its effects on the serum lipid levels in metabolic syndrome patients. 2016;54(6):894-902. 
35. Freeman DJ, Griffin BA, Holmes AP, Lindsay GM, Gaffney D, Packard CJ, et al. Regulation of plasma HDL cholesterol and subfraction distribution by genetic and environmental factors. Associations between the Taql B RFLP in the CETP gene and smoking and obesity. 1994;14(3):336-44.

36. Vohl M-C, Lamarche B, Pascot A, Leroux G, Prud'homme D, Bouchard C, et al. Contribution of the cholesteryl ester transfer protein gene TaqlB polymorphism to the reduced plasma HDL-cholesterol levels found in abdominal obese men with the features of the insulin resistance syndrome. 1999;23(9):918-25.

37. Asayama K, Hayashibe H, Dobashi K, Uchida N, Nakane T, Kodera K, et al. Increased serum cholesteryl ester transfer protein in obese children. 2002;10(6):439-46.

38. Lassale C, Fezeu L, Andreeva V, Hercberg S, Kengne A, Czernichow S, et al. Association between dietary scores and 13-year weight change and obesity risk in a French prospective cohort. 2012;36(11):1455-62.

39. Bruun JM, Stallknecht B, Helge JW, Richelsen BJEjoe. Interleukin-18 in plasma and adipose tissue: effects of obesity, insulin resistance, and weight loss. European Journal of Endocrinology. 2007;157(4):465-71.

40. Styskal J, Van Remmen H, Richardson A, Salmon ABJFRB, Medicine. Oxidative stress and diabetes: what can we learn about insulin resistance from antioxidant mutant mouse models? 2012;52(1):46-58.

41. Maciejczyk M, Żebrowska E, Zalewska A, Chabowski AJOm, longevity c. Redox balance, antioxidant defense, and oxidative damage in the hypothalamus and cerebral cortex of rats with high fat diet-induced insulin resistance. 2018;2018.

42. Gammon CS, Minihane AM, Kruger R, Conlon CA, von Hurst PR, Jones B, et al. TaqlB polymorphism in the cholesteryl ester transfer protein (CETP) gene influences lipid responses to the consumption of kiwifruit in hypercholesterolaemic men. Br J Nutr. 2014;111(6):1077-84.

43. Yaghoubi A, Ghojazadeh M, Abolhasani S, Alikhah H, Khaki-Khatibi F. Correlation of Serum Levels of Vitronectin, Malondialdehyde and Hs- CRP With Disease Severity in Coronary Artery Disease. J Cardiovasc Thorac Res. 2015;7(3):113-7.

44. Du J, Fang DZ, Lin J, Xiao LY, Zhou XD, Shigdar S, et al. TaqIB polymorphism in the CETP gene modulates the impact of HC/LF diet on the HDL profile in healthy Chinese young adults. J Nutr Biochem. 2010;21(11):1114-9.

45. Physical inactivity and excessive sucrose consumption are associated with higher serum lipids in subjects with Taq1B CETP polymorphism.

46. Riemens SC, van Tol A, Sluiter WJ, Dullaart RP. Plasma phospholipid transfer protein activity is lowered by 24-h insulin and acipimox administration: blunted response to insulin in type 2 diabetic patients. Diabetes. 1999;48(8):1631-7.

47. Siewert S, Gonzalez, II, Lucero RO, Ojeda MS. Association of cholesteryl ester transfer protein genotypes with paraoxonase-1 activity, lipid profile and oxidative stress in type 2 diabetes mellitus: A study in San Luis, Argentina. J Diabetes Investig. 2015;6(1):67-77.

48. Russo GT, Horvath KV, Di Benedetto A, Giandalia A, Cucinotta D, Asztalos BJA. Influence of menopause and cholesteryl ester transfer protein (CETP) TaqIB polymorphism on lipid profile and HDL subpopulations distribution in women with and without type 2 diabetes. 2010;210(1):294-301.

49. Rezazadeh K, Rezazadeh F, Ebrahimi-Mameghani MJEJoIM. The effect of artichoke leaf extract supplementation on lipid and CETP response in metabolic syndrome with respect to Taq 1B CETP polymorphism: A randomized placebo-controlled clinical trial. 2018;17:112-8.

50. Noorshahi N, Sotoudeh G, Djalali M, Eshraghian MR, Keramatipour M, Basiri MG, et al. APOA II genotypes frequency and their interaction with saturated fatty acids consumption on lipid profile of patients with type 2 diabetes. Clinical nutrition (Edinburgh, Scotland). 2016;35(4):907-11.

51. Puchau B, Zulet M, Echávarri A, Hermsdorff HH, Alfredo M. Dietary total antioxidant capacity is negatively associated with some metabolic syndrome features in healthy young men. Nutrition (Burbank, Los Angeles County, Calif). 2009;26:534-41.

52. Dorosti Motlagh AR TM. Iranain Food Composition Table. Iran Donyaye Taghzieh Press;. 2007.

53. Esfahani FH, Asghari G, Mirmiran P, Azizi F. Reproducibility and relative validity of food group intake in a food frequency questionnaire developed for the Tehran Lipid and Glucose Study. Journal of epidemiology. 2010;20(2):150-8.

54. Holt SH, Miller JC, Petocz P. An insulin index of foods: the insulin demand generated by 1000-kJ portions of common foods. The American journal of clinical nutrition. 1997;66(5):1264-76.

55. Bao J, de Jong V, Atkinson F, Petocz P, Brand-Miller JC. Food insulin index: physiologic basis for predicting insulin demand evoked by composite meals. The American journal of clinical nutrition. 2009;90(4):986-92.

56. Nimptsch K, Brand-Miller JC, Franz M, Sampson L, Willett WC, Giovannucci E. Dietary insulin index and insulin load in relation to biomarkers of glycemic control, plasma lipids, and inflammation markers. The American journal of clinical nutrition. 2011;94(1):182-90.

57. Alvandi E, Akrami SM, Chiani M, Hedayati M, Nayer BN, Tehrani MR, et al. Molecular analysis of the RET proto-oncogene key exons in patients with medullary thyroid carcinoma: a comprehensive study of the Iranian population. Thyroid : official journal of the American Thyroid Association.

2011;21(4):373-82.

\section{Tables}

Table1: The association between baseline characteristic and metabolic markers with Dietary Insulin Load(DIL) and Dietary Insulin Index (DII) in T2DM patients 


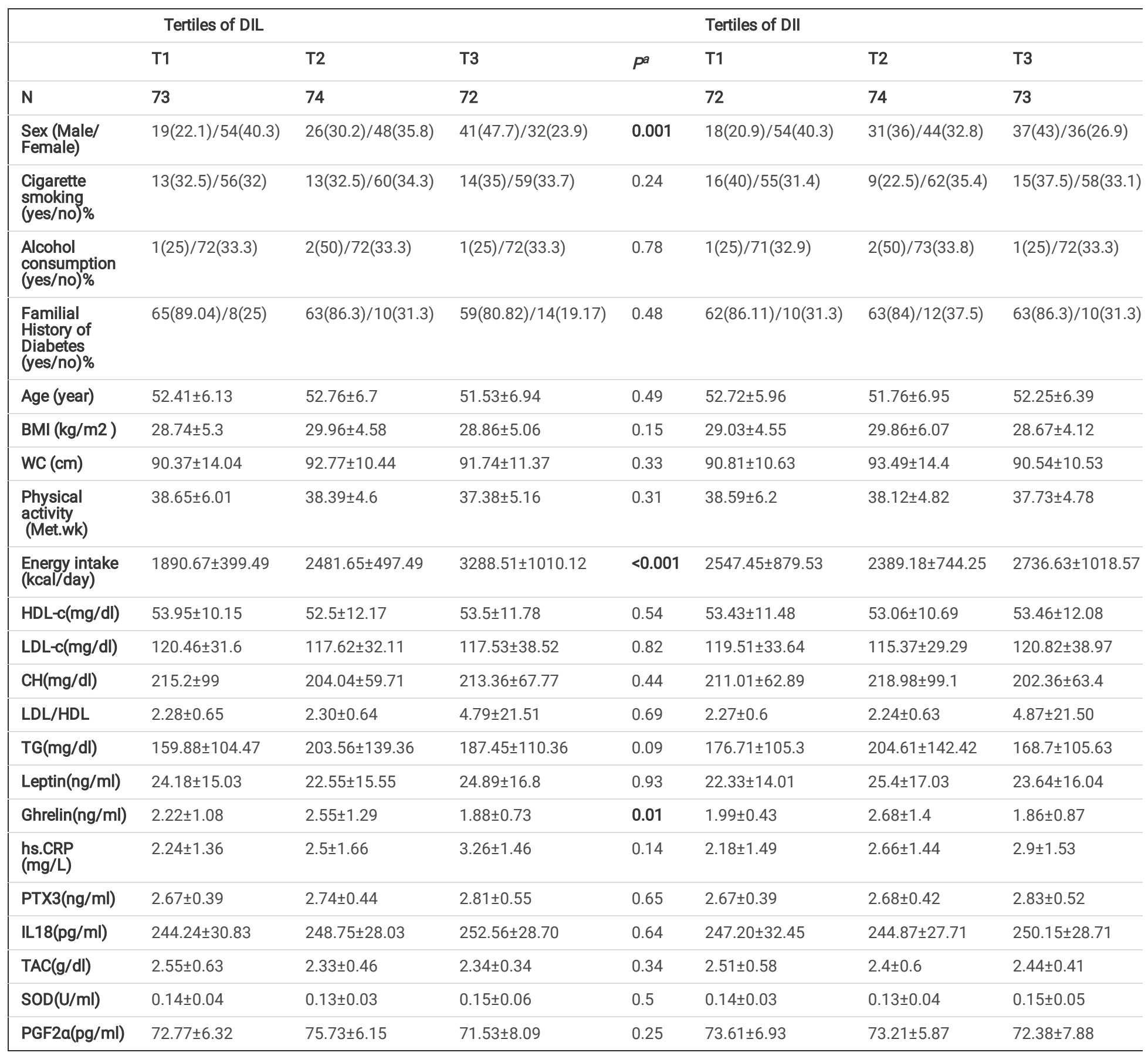

Data are presented as mean \pm standard deviation (SD) or percent. Abbreviation: DIL: dietary insulin load, DII: dietary insulin index, BMI: body mass index, HDLc high density lipoprotein cholesterol, $\mathrm{LDL}-\mathrm{c}=$ low density lipoprotein cholesterol, $\mathrm{CH}=$ cholesterol, $\mathrm{TG}=$ triglyceride, $\mathrm{CRP}=\mathrm{C}$-reactive protein, $\mathrm{PTX} 3=\mathrm{Pentraxin}$ 3, IL18 = interleukin 18, TAC = total antioxidant capacity, SOD = superoxide dismutase, PGF2a = prostaglandinF2a ${ }^{\text {a }}$ Obtained from ANOVA or Chi-square test, where appropriate

\section{Figures}



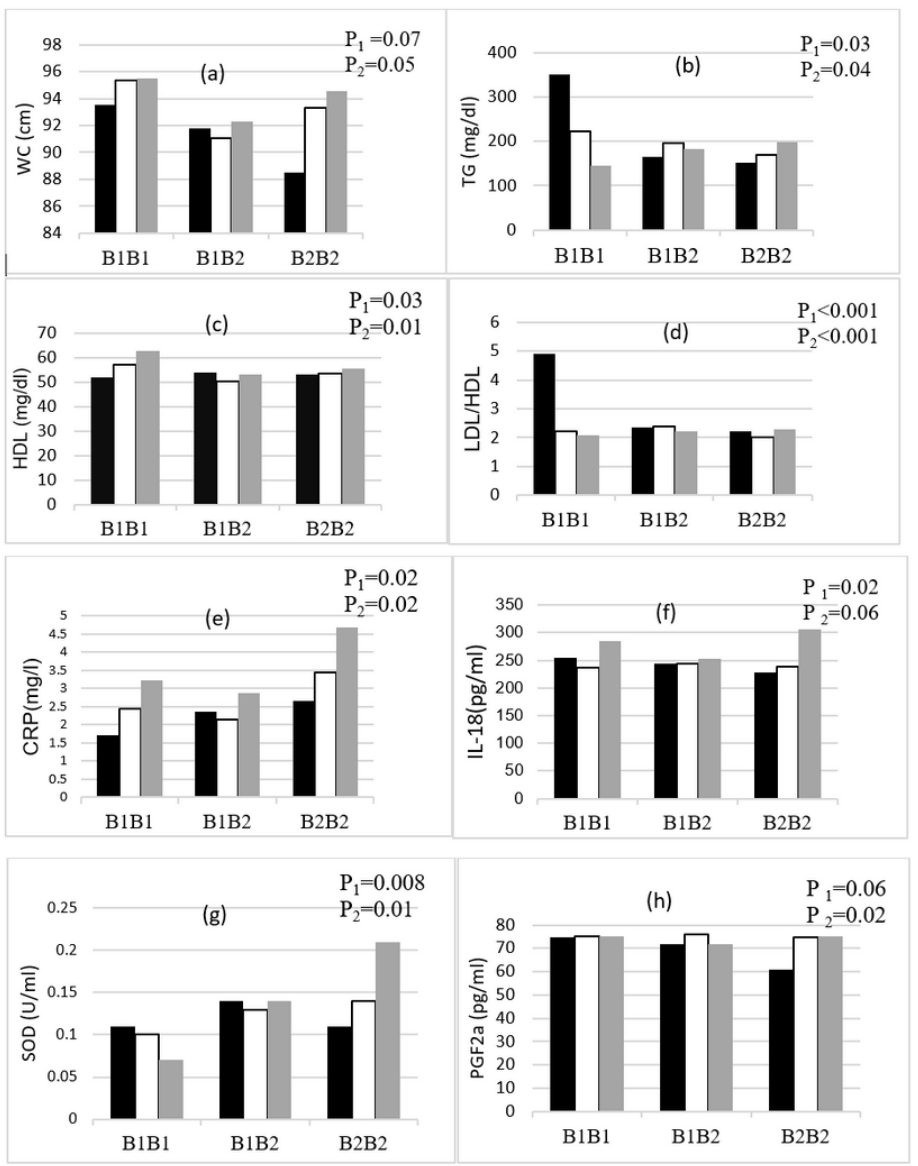

$\mathrm{P}_{1}=\mathrm{P}$-value for curd model

First tertile of DIL

$\mathrm{P}_{2}=\mathrm{P}$-value for the adjusted model by age, gender, physical activity, smoking, alcohol consumption, and familial history of diabetes

$\square$ Second tertile of DIL

$\square_{\text {Third tertile of DIL }}$

\section{Figure 1}

(a) = Mean values of WC across CETP genotypes (B1 B, B1B2, B2B2) based on low and high DIL intake (b): Mean values of TG across CETP genotypes (B1 B, B1B2, B2B2) based on low and high DIL intake (C): Mean values of HDL across CETP genotypes (B1B1, B1B2, B2B2) based on low and high DIL intake (d): Mean values of LDL/HDL across CETP genotypes (B1B1, B1B2, B2B2) based on low and high DIL intake (e): Mean values of CRP across CETP genotypes (B1 B1, B1B2, B2B2) based on low and high DIL intake (f): Mean values of IL-18 across CETP genotypes (B1B1, B1B2, B2B2) based on low and high DIL intake (g): Mean values of SOD across CETP genotypes (B1B1, B1B2, B2B2) based on low and high DIL intake (h): Mean values of PGF2a across CETP genotypes (B1B1, B1B2, B2B2) based on low and high DIL intake 

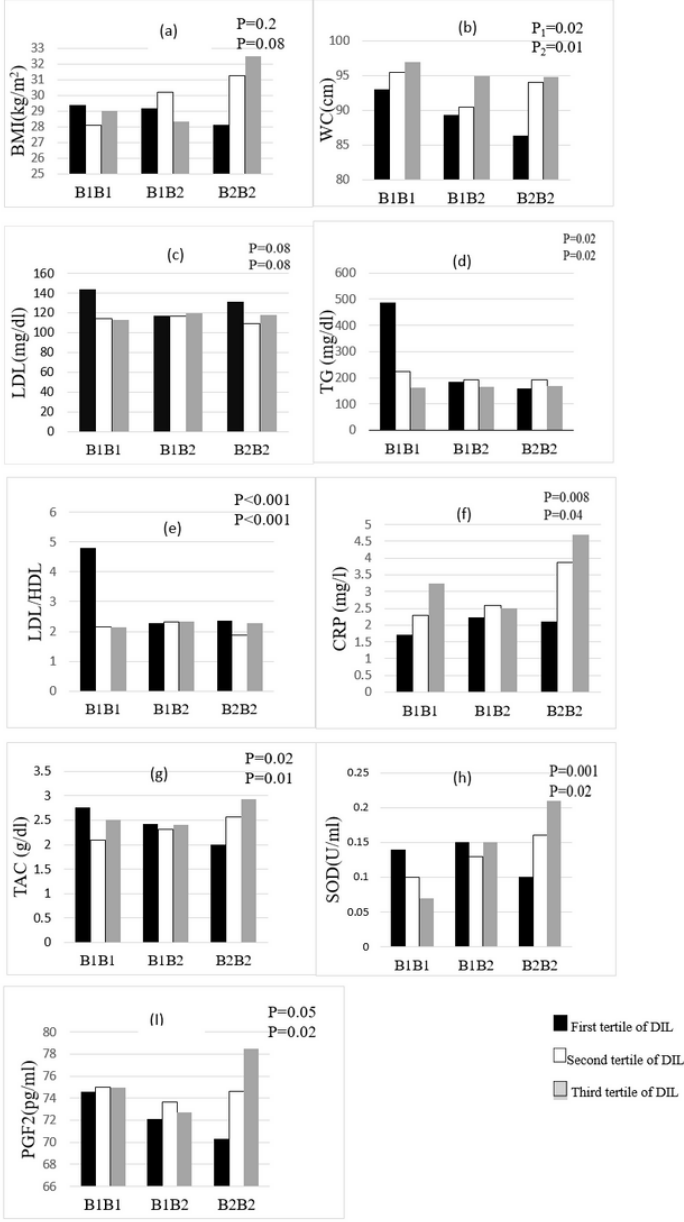

$\mathrm{P}_{1}=\mathrm{P}$-value for curd model

$\mathrm{P}_{2}=\mathrm{P}$-value for the adjusted model by age, gender, physical activity, smoking, alcohol consumption, and
familial history of diabetes

\section{Figure 2}

(a) = Mean values of BMI across CETP genotypes (B1B1, B1B2, B2B2) based on low and high DIl intake (b): Mean values of WC across CETP genotypes (B1B1, B1B2, B2B2) based on low and high DII intake (C): Mean values of LDL across CETP genotypes (B1B1, B1B2, B2B2) based on low and high DII intake (d): Mean values of TG across CETP genotypes (B1B1, B1B2, B2B2) based on low and high DIl intake (e): Mean values of LDL/HDL across CETP genotypes (B1B1, B1B2, B2B2) based on low and high DIl intake (f): Mean values of CRP across CETP genotypes (B1B1, B1B2, B2B2) based on low and high DII intake (g): Mean values of TAC across CETP genotypes (B1B1, B1B2, B2B2) based on low and high DII intake (h): Mean values of SOD across CETP genotypes (B1B1, B1B2, B2B2) based on low and high DIl intake (I): Mean values of PGF2a across CETP genotypes (B1B1, B1B2, B2B2) based on low and high DII intake 